\title{
Trypanosuppressive effects of Kolaviron may be associated with down regulation of Trypanothione reductase in Trypanosoma congolense infection
}

\author{
Timothy, M.R. ${ }^{1,4}$, Ibrahim, Y.K.E. ${ }^{2,4}$, Muhammad, A. ${ }^{1,4^{*}}$, Chechet, G.D. ${ }^{1,4}$, Aimola, I.A. ${ }^{1,4}$, Mamman, M. ${ }^{3,4}$ \\ ${ }^{1}$ Department of Biochemistry, Faculty of Life Sciences, Ahmadu Bello University, Zaria, Kaduna State, Nigeria \\ ${ }^{2}$ Faculty of Pharmaceutical Sciences, Ahmadu Bello University, Zaria, Kaduna State, Nigeria \\ ${ }^{3}$ Department of Veterinary Pharmacology and Toxicology, Faculty of Veterinary Medicine, Ahmadu Bello University, Zaria, Kaduna State, Nigeria \\ ${ }^{4}$ African Centre of Excellence for Neglected Tropical Diseases and Forensic Biotechnology, Ahmadu Bello University Centre, Zaria, Nigeria \\ *Corresponding author: amachida31@gmail.com
}

\section{ARTICLE HISTORY}

Received: 24 April 2019

Revised: 11 April 2020

Accepted: 16 April 2020

Published: 25 March 2021

\begin{abstract}
Trypanothione reductase is a key enzyme that upholds the redox balance in hemoflagellate protozoan parasites such as $T$. congolense. This study aims at unraveling the potency of Kolaviron against trypanothione reductase in $T$. congolense infection using Chrysin as standard. The experiment was performed using three different approaches; in silico, in vitro and in vivo. Kolaviron and Chrysin were docked against trypanothione reductase, revealing binding energies $(-9.3$ and $-9.0 \mathrm{kcal} / \mathrm{mol})$ and $\mathrm{K}_{\mathrm{i}}$ of $0.211 \mu \mathrm{M}$ and $0.151 \mu \mathrm{M}$ at the active site of trypanothione reductase as evident from the observed strong hydrophobic/hydrogen bond interactions. Parasitized blood was used for parasite isolation and trypanothione reductase activity assay using standard protocol. Real-time PCR ( $(\mathrm{PPCR}$ ) assay was implored to monitor expression of trypanothione reductase using primers targeting the 177-bp repeat satellite DNA in $T$. congolense with SYBR Green to monitor product accumulation. Kolaviron showed $I_{50}$ values of $2.64 \mu \mathrm{g} / \mathrm{ml}$ with $\%$ inhibition of 66.78 compared with Chrysin with $\mathrm{IC}_{50}$ values of $1.86 \mu \mathrm{g} / \mathrm{ml}$ and $\%$ inhibition of 53.80 . In vivo studies following the administration of these compounds orally after 7 days post inoculation resulted in \% inhibition of Chrysin (57.67) and Kolaviron (46.90). Equally, Kolaviron relative to Chrysin down regulated the expression trypanothione reductase gene by 1.352 as compared to 3.530 of the infected group, in clear agreement with the earlier inhibition observed at the fine type level. Overall, the findings may have unraveled the Kolaviron potency against Trypanosoma congolense infection in rats.
\end{abstract}

Keywords: Trypanothione reductase; Kolaviron; Chrysin; Inhibition; Expression.

\section{INTRODUCTION}

Parasitic diseases are a major obstacle to both human and animal health with substantial economic losses by affecting livestock (Kumar et al., 2014). Trypanosomiasis is a neglected African tropical disease caused by a unicellular, flagellated protozoan parasite belonging to the genus Trypanosoma (Chanie et al., 2018). They are transmitted to both human and animals by tsetse fly bite (Glossina spp.) (Peacock et al., 2012). This parasite has a complex life cycle and is divided into two phases, one in the tsetse fly vector and the other in the bloodstream of the mammalian host (Barrett et al., 2007; Baral, 2010). The species responsible for Human African Trypanosomiasis (HAT) also known as sleeping sickness are $T$. brucei gambiense and T. brucei rhodesiense while T. congolense, $T$. vivax, $T$. evansi and $T$. equiperdum are responsible for animal trypanosomiasis (AT) also known as "Nagana" in cattle and "Surra" in camel (Baker et al., 2013).

The chemotherapy for the the treatment of sleeping sickness is suramin and pentamidine in the early stage whereas eflornithine and melarsoprol are used in the latestage. The efficacy of these chemotherapeutics is associated with several setbacks such as unavailability, toxicity and emergence of widespread drug resistance (Holloway et al., 2009; Baker et al., 2013). Thus, there is an urgent need to discover new compounds as starting point for the development of potent, more efficient drug with fewerside effects, preferably interfering with unique essential pathways of these parasites (Beig et al., 2015). Thus, one of the priorities in tropical medicine research has been the identification and characterisation of parasite-specific biomolecules, which play relevant physiological roles and thus might be exploited as selective targets (Tovar et al., 2006).

The promising target for the design of a new drug involves thiol metabolism in these protozoans where redox balance is maintained uniquely by the dithiol Trypanothione (Beig et al., 2015). Trypanothione $\left[N^{1}, N^{8}\right.$-bis (glutathionyl) spermidine counteracts environmental oxidative stress through a variety of enzymatic and non-enzymatic reactions, and has been implicated in acquired resistance to chemotherapeutic 
agents (Bailey et al., 1993). Most of these protective reactions oxidize trypanothione $\mathrm{T}[\mathrm{SH}]_{2}$ to trypanothione disulfide $\mathrm{T}[\mathrm{S}]_{2}$, which is recycled back to $\mathrm{T}[\mathrm{SH}]_{2}$ by trypanothione reductase (TryR; EC 1.8.1.12), an NADPH-dependent disulphide oxidoreductase. Trypanothione reductase (TryR) is thought to be the central enzyme in the redox metabolism of these protozoans, being the sole route of reducing equivalents from the $\mathrm{NADP}^{+} / \mathrm{NADPH}$ couple to thiol-containing species (Beig et al., 2015).

Trypanothione reductase (TryR) and human glutathione reductase (GR) have comparable catalytic reactions whereby 14 of the 19 amino acid residues near to the binding site are evolutionarily conserved. This is because; the nearest homologue of TryR in Human cells is GR with approximately $40 \%$ sequence homology. Conversely, they are specific to their individual disulphide substrates. GR has a hydrophilic, positively charged region in its active site that interrelates with the glycine carboxylates of glutathione disulfide, whereas TryR has a superior binding site, with a hydrophobic and negatively charged region with which the spermidine moiety of trypanothione disulphide binds. The absence of this enzyme from the mammalian host and the sensitivity of trypanosomatids to oxidative stress makes it a striking target for trypanosomiasis therapeutics (Saha \& Sharma, 2015), in addition to superoxide dismutase and peroxidases which are not trypanosome-specific.

Oxidative stress is an imbalance between production of free radical and the reactive metabolite (antioxidants). Disturbance in this normal redox state through blocking of the enzyme active site by selective inhibition can cause toxic effect through the accumulation of peroxides and free radicals with a resultant oxidative stress on the cell which damages the cell components of the parasite (Adaramoye \& Arisekola, 2013). Hence, the use of flavonoids as potential inhibitors cannot be overemphasised. Kolaviron and Chrysin are examples of flavonoids (secondary metabolite) that are found in fruits, vegetables, honey and beverages (Gupta et al., 2014). Flavonoids exhibit wide range of biological activities such as anti-inflammatory (Dixit, 2014), antimicrobial, anti-allergic and anti-oxidants effects (Farombi et al., 2013; Kaidama \& Gacche, 2015). They have also demonstrated in vivo and in vitro leishmanicidal, trypanocidal, antioxidant, and prooxidant properties (Baldim et al., 2017). Kolaviron is a biflavonoid of Garcinia kola seeds with reported antifilarial property (Muhammad et al., 2017), however, its efficacy on Trypanosoma congolense not yet been reported. Even though other flavonoids like Chrysin have so far been tested against trypanosomes such as Trypanosoma brucei (Tasdemir et al., 2006; Baldim et al., 2017), use of these compounds (Kolaviron and Chrysin) especially for the purpose of targeting TryR in Trypanosoma congolense infection is yet to be explored. More importantly, Chrysin can thus be harnessed here since its efficacy has been previously reported against Trypanosoma brucei (Baldim et al., 2017), and was therefore used as standard in the current study. Accordingly, present study, for the first time, aims to explore the potential of Kolaviron in the amelioration of trypanosome infection by focusing on its possible inhibitory activity against trypanothione reductase and down regulation of the gene that expresses it.

\section{MATERIALS AND METHODS}

\section{Molecular modelling of Trypanothione Reductase}

The structure of the ligands (Figures 1-2) Chrysin (5281607) and Kolaviron (155169) were retrieved from major ligand data base, PubChem (Figures 1-2). A PDB format of the target protein; trypanothione reductase (4NEV) was obtained from protein data bank. The protein preparation and energy minimization were done using Discovery studio $2.5 \mathrm{v}$. A sphere binding site with $9.0 \AA$ radius was defined around the bounded ligand to identify the binding site of the protein structure. The CHARMm-based DOCKER program was used to score the interaction between the protein and the ligands (Chrysin and Kolaviron) into the crystal structure of the receptor binding pocket. The PDB files of the best docked conformation were generated using Accerlrys discovery studio. This was followed by importation into Chimera $1.1 \mathrm{v}$ to view the hydrophobic and hydrophilic interactions. The binding energies and kinetic binding inhibition constants were equally generated.

\section{Extraction of Kolaviron}

Kolaviron was extracted in the Drug metabolism and Molecular Toxicology Laboratory Department of Biochemistry, University of Ibadan, Ibadan, Nigeria using the method as previously described (Olaleye et al., 2000), from fresh Garcinia kola seeds. The powdered seed $(600 \mathrm{~g})$ was defatted with $800 \mathrm{ml}$ of light petroleum ether (BP $40-60^{\circ} \mathrm{C}$ ) in a Soxhlet apparatus for $24 \mathrm{~h}$. The defatted sample was spread in thin layers on trays and air dried at room temperature for $24 \mathrm{~h}$, and thereafter repacked in the Soxhlet apparatus and reextracted with acetone $(500 \mathrm{ml})$ at a temperature of $40^{\circ} \mathrm{C}$. The

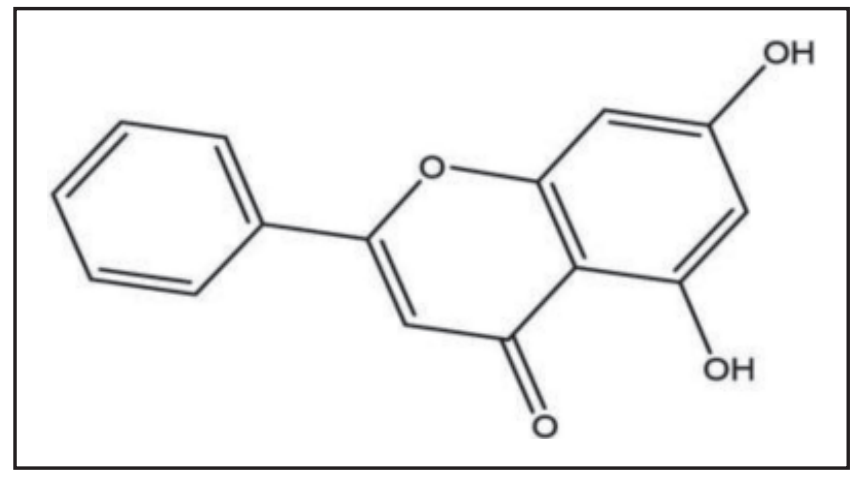

Figure 1. Structure of Chrysin.

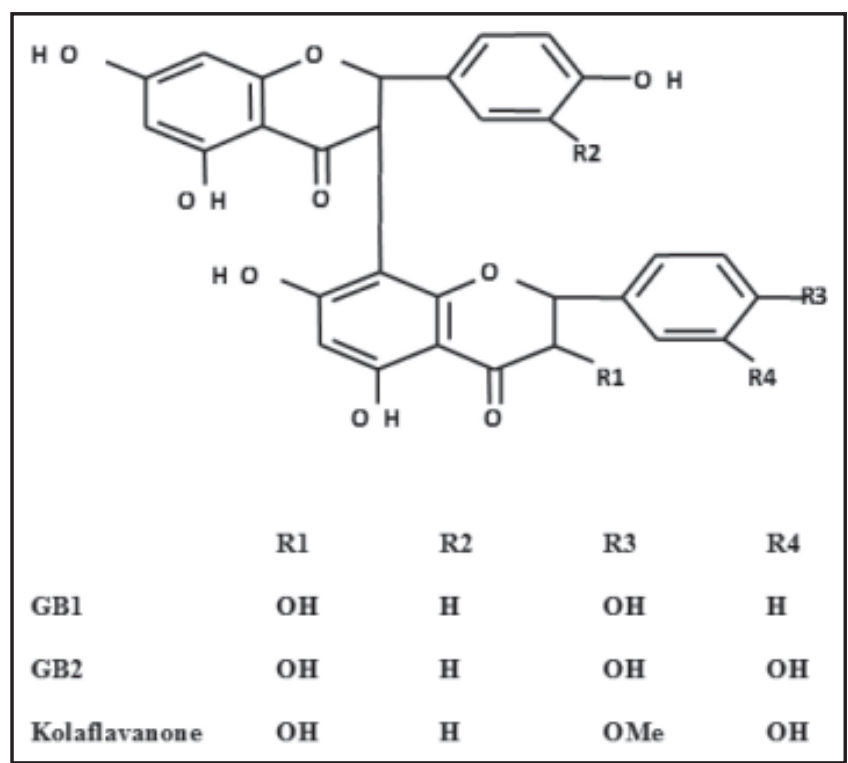

Figure 2. Structure of Kolaviron. 
extract was concentrated and diluted twice its volume with distilled water and extracted with ethyl acetate (6:3). The concentrated ethyl acetate extract yields a yellow solid known as Kolaviron. Kolaviron was separated by thin layer chromatography (TLC) using silica gel GF 254 coated plates and solvent mixture chloroform/methanol (80:20). TLC revealed the presence of three compounds GB1, GB2 and Kolaflavanone in a ratio 2:2:1 which was identified by their $R_{\mathrm{f}}$ values compared with reference compounds.

\section{Parasite}

Trypanosoma congolense strain (molecularly characterised to confirm its identity using ITS primer with a band size of $640 \mathrm{bp})$ was obtained from Nigerian Institute for Trypanosomiasis Research (NITR), Kaduna. The parasite was maintained by serial passage in donor rats.

\section{Experimental animals and design for in vitro and in vivo studies} Thirty-five (35) male Wistar rats were used for this study. They were acclimatized for 2 weeks under standard laboratory condition in the Faculty of Pharmaceutical Sciences animal house before commencement of the experiment which lasted for 4 weeks. The rats were randomly grouped into seven groups of five each. The experiment was carried out in two stages; stage 1 , in vitro study and stage 2 , in vivo which lasted for 2 weeks each. In any case, ethical clearance was obtained from Animal rights review board of Ahmadu Bello University Zaria, Kaduna State, Nigeria.

In stage 1: Five Wistar rats were infected with the parasite and daily parasitaemia levels were monitored. At peak parasitaemia $\left(10^{7}\right)$, the rats were sacrificed, and the blood was collected. This was followed by parasite isolation for the in vitro study.

In stage 2: The treatment was orally administered as follows:

Group 1: Infected with the parasite and treated with the Chrysin $(40 \mathrm{mg} / \mathrm{kg} / \mathrm{b} . \mathrm{w})$ (Kaidama \& Gacche, 2015). This is to serve as reference pure and synthetic flavonoid as reported in a previous study with Trypanosoma brucei (Tasdemir et al., 2006; Baldim et al., 2017) and has structural analogy with Kolaviron.

Group 2: Infected with the parasite and treated with the Kolaviron (200mg/kg/b.w.) (Olaleye et al., 2000).

Group 3: Infected with the parasite with no treatment (serving as control).

It is however, important to note that the selected doses (in vitro and in vivo) of Kolaviron and Chrysin were based on their reported anti-inflammatory and antioxidant activities visà-vis our subsequent pilot studies conducted before the commencement of the main experiments.

\section{Parasite inoculation of Wistar rats}

Parasitized blood was obtained from the tail of rats displaying a parasitaemia of $1 \times 10^{7}$ Trypanosomes $/ \mathrm{ml}$ and was maintained in normal saline solution which was used to inoculate the rats. The suspension $(0.3 \mathrm{ml})$ containing 3-4 trypanosome per field at a magnification of $\times 400$ as previously described (Tasdemir et al., 2006), was used to infect the rats through the intra-peritoneal cavity of the uninfected rat.

\section{Parasite collection/ purification}

Blood was collected from infected rats at peak parasitaemia seven days post infection, for the in vitro studies using the rapid matching method (Herbert and Lumsden, 1976). The rats were sacrificed by decapitation following mild chloroform anesthesia using American Veterinary Medical Association (AVMA) Guidelines for the Euthanasia of Animals, 2013 Edition. Parasites were isolated from the blood according to method of Godfrey and Lanham (Godfrey \& Lanham, 1971). Accordingly, the blood was carefully introduced into the column on the packed gel that allows moving down the stationary phase. The blood being negatively charged was bound to the gel matrix while the parasites were eluted and collected in fractions of $0.5 \mathrm{ml}$.

\section{In vitro and In vivo Enzyme assays}

The trypanothione reductase (TryR) activity was assayed spectrophotometrically at 410nm (Holloway et al., 2009) after a prewash with phosphate-buffered saline to remove potentially interfering compounds. Exactly $5 \mathrm{ml}$ of the isolated parasite was chemically lysed by 30 min incubation with a lysing buffer $(200 \mu \mathrm{l} /$ well), consisting of EDTA (1mM), HEPES (40mM), Tris (50mM; pH 7.5), and Triton X-100 (2\% v/v). Immediately prior to use, the buffer was supplemented with the protease inhibitor phenylmethanesulfonyl fluoride at a concentration of $1 \mathrm{mM}$. TryR activity was measured in $100 \mu \mathrm{l}$ of sample lysate, dispensed into the test well of a 96-well plate. NADPH $(50 \mu \mathrm{l} /$ well), Trypanothione disulphide $(100 \mu \mathrm{l} /$ well), was sequentially added to the sample lysate. A blank was set for each sample, consisting of sample lysate supplemented with the reaction mixture described above, in which the substrate Trypanothione disulphide was replaced by $(0.05 \mathrm{M})$ Tris buffer, $\mathrm{pH}$ 7.5. After a serial $27^{\circ} \mathrm{C}$ incubation ranging from 1 minute to 30 minutes, absorbance was measured with a spectrumlab $22 \mathrm{c}$ at a wavelength of $410 \mathrm{~nm}$. A Serial dilution of the stock solution of the compounds (Kolavrion and Chrysin); 10, 20, 40 and $80(\mu \mathrm{g} / \mathrm{ml})$, was done using distilled water as previously described (Umar et al., 2010). Assessment of the in vitro inhibition activity of the compounds on the enzyme trypanothione reductase was performed in triplicates and $\mathrm{IC}_{50}$ was determined as previously described (Malele et al., 2003).

For in vivo evaluation, Wistar rats were infected intraperitoneally and the level of parasitaemia was monitored microscopically at $\times 400$ using the method of Herbert and Lumsden (Herbert \& Lumsden, 1976). Doses of Chrysin and Kolaviron as described in previous section were administered orally for the period of 12 days post infection, while parasitaemia level was monitored as also previously described. Twenty-four hours after the last treatment, the rats were sacrificed, and the blood was collected. The parasites were isolated, lysed and the enzyme activity was determined as described in the previous section above. The $\%$ inhibition was also determined.

\section{RNA extraction/ quantification}

Total RNA was isolated using, Accuprep Viral RNA extraction kit (Bioneer USA) according to the manufacturer's instructions. Exactly $200 \mathrm{ml}$ of parasite suspension was dispensed into a $1.5 \mathrm{ml}$ eppendorf tube; $400 \mathrm{ul}$ binding buffer was added in the tube and mixed by lightly vortexed for 5 seconds for efficient lysing. The mixture was incubated for 10 minutes at room temperature. A volume of $100 \mathrm{ml}$ of isopropanol was added and lightly vortexes for 5 seconds, the mixture was spun down for 10 seconds. The binding column was fit into $2 \mathrm{ml}$ collection tube while the liquid was transferred into the binding column. The lid was carefully closed and centrifuged for 1 minute at $8,000 \mathrm{rpm}$ for the liquid to completely pass through the column following centrifugation, thereafter the binding column was transferred to a new $2 \mathrm{ml}$ collection tube after centrifugation. The binding column was 
transferred to $2 \mathrm{ml}$ collection tube, $500 \mathrm{ml}$ of $\mathrm{W} 2$ buffer added and centrifuged for 1 minute at $8,000 \mathrm{rpm}$. The mixture was spun down once more at $13,000 \mathrm{rpm}$ for 1 minute to remove ethanol completely. The binding column was transferred to a $1.5 \mathrm{ml}$ collection tube, $50 \mathrm{ml}$ of Elution Buffer was added and left to stand for 1 minute to allow the buffer to permeate the column. The RNA was eluted by spinning down at 8,000 rpm for 1 minute. The eluted RNA solution was immediately used or stored at $-80^{\circ} \mathrm{C}$ for further analysis after quantification at appropriate wavelength of maximum absorption. The purity was also ascertained.

\section{Complementary DNA (cDNA) synthesis}

The isolated RNA was transcribed into complementary DNA using the cDNA synthesis kit following the manufacturer's instruction (Bioneer, Accuprep, USA). In a $20 \mu$ l reverse transcriptase reaction mixture, the RNA samples were mixed with the primer $(18 \mu)$ of the RNA template and $2 \mu$ l of the random hexamer) for 1 cycle at $70^{\circ} \mathrm{C}$ for $5 \mathrm{~min}$ in a PCR mastercycler. Reaction buffer, RNAse inhibitor and DNTPs were added. The following cycle conditions were used: $30^{\circ} \mathrm{C}$ for $5 \mathrm{~min}, 42^{\circ} \mathrm{C}$ for $60 \mathrm{~min}$ and $94^{\circ} \mathrm{C}$ for $5 \mathrm{~min}$. At the end of the incubation, samples were diluted and stored at $-20^{\circ} \mathrm{C}$ for further analysis.

\section{Quantitative Real-time PCR}

Quantitative real-time polymerase chain reaction (qPCR) was performed using light cycler system (version 3.5 Roche, Basel Switzerland). Each sample was tested in triplicate and GAPDH was used as internal control. Forward and reverse primers used for GAPDH were 5'-CTCGGCGTTGAGTACGTGAT-3' and 5'-GCTCGCGTGGGTTATAGTCA-3', whereas those of Try $R$ gene were $5^{\prime}$-GTTGCAGACTGTCGGAGTAAA-3' and 5'-CAGCATAA TACGACCCGTTACA-3', respectively. In a $20 \mu$ l reaction volume containing $12 \mu \mathrm{l}$ ready to use SYBR Green 1 master mix, $5 \mu$ l of the cDNA transcribed, $1 \mu \mathrm{l}$ of forward and reverse primer each, and $1 \mu$ of RNase free water were added. The thermocycler condition consisted of $1 \mathrm{cycle}$ at $94^{\circ} \mathrm{C}$ for $15 \mathrm{~min} 62^{\circ} \mathrm{C}$ for $15 \mathrm{~s}$, and $72^{\circ} \mathrm{C}$ for $30 \mathrm{~s}$ was strictly followed. A melting curve was obtained for each quantitative PCR run and the second derivative maximum method was used to determine the crossing point $(C p)$ for individual samples. The real-time PCR data were analysed using the $2^{-\Delta \Delta C T}$ relative quantization method following the manufacturer's instructions.

\section{Data Analysis}

To address variability, experiments were repeated at least three times. Where appropriate, data were presented as Mean \pm Standard Deviation. Variability between groups was measured using one-way ANOVA by Statistical Package for Social Sciences Software (SPSS) version 20.0 (SPSS Inc., Chicago, Illinois, USA). Level of significance was measured using least significant difference (LSD). $\mathrm{P}<0.05$ was considered statistical significant.

\section{RESULTS}

Effects of Chrysin and Kolaviron on Trypanothione Reductase in silico

Table 1 shows the Binding Energy of ligands (Chrysin and Kolaviron) after docking with trypanothione reductase (Figures 3-4). The values are Chrysin $(-9.3 \mathrm{kcal} / \mathrm{mol})$ and Kolaviron $(-9.0 \mathrm{kcal} / \mathrm{mol})$, respectively. Because of this finding, Chrysin and Kolaviron were seemed to be potential candidates for in vitro studies.

Table 1. The Binding Energies/Inhibition Binding Constant for Chrysin and Kolaviron against Trypanothione reductase in silico

\begin{tabular}{lccc}
\hline S/No & Ligand & Binding Energy $(\mathrm{Kcal} / \mathrm{mol})$ & $\mathrm{K}_{\mathrm{i}}(\mu \mathrm{M})$ \\
\hline 1. & Chrysin & -9.30 & 0.15 \\
2. & Kolaviron & -9.00 & 0.21 \\
\hline
\end{tabular}

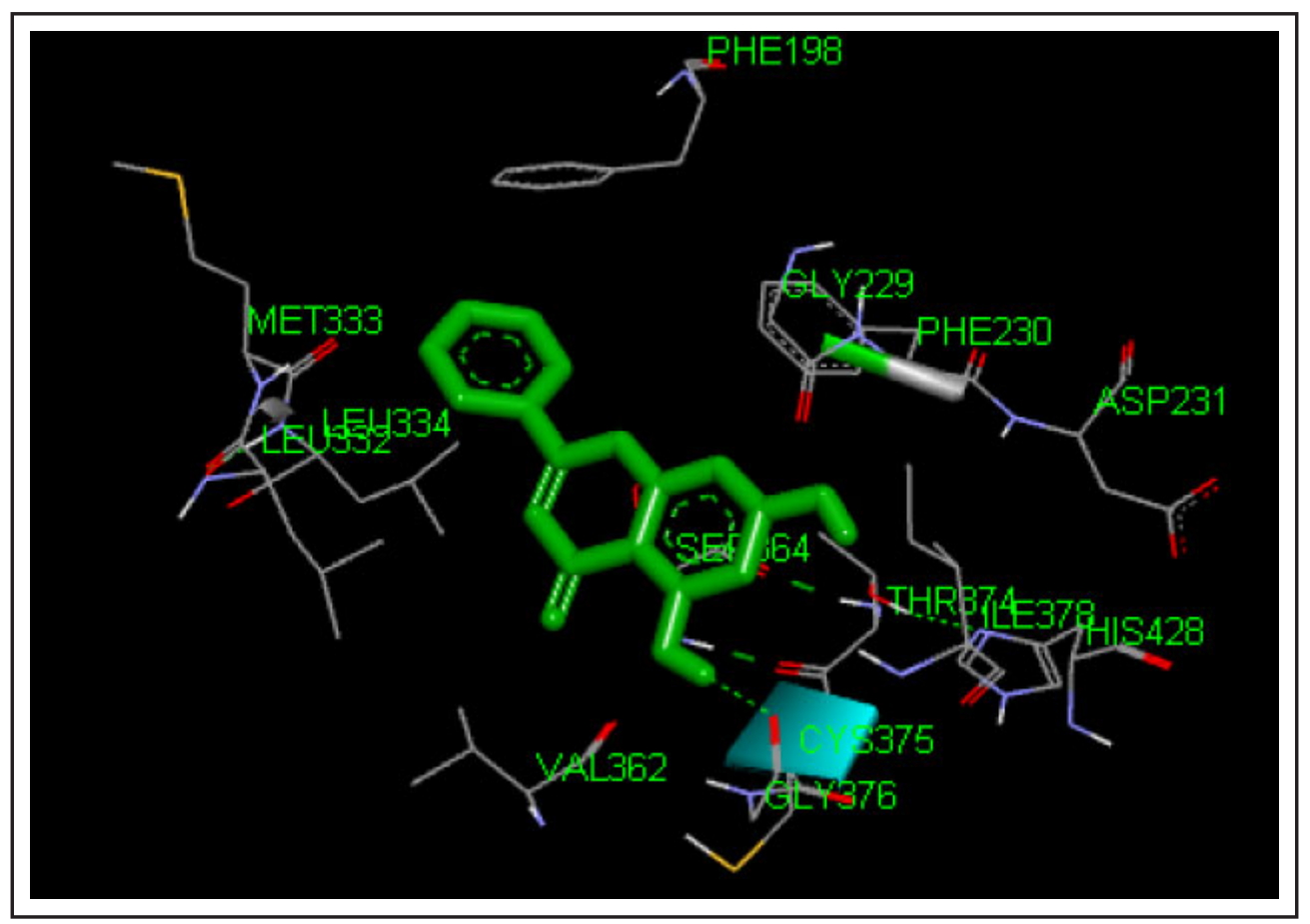

Figure 3. 2D Schematic presentation of the active site of Trypanothione reductase and ligand (Chrysin). 


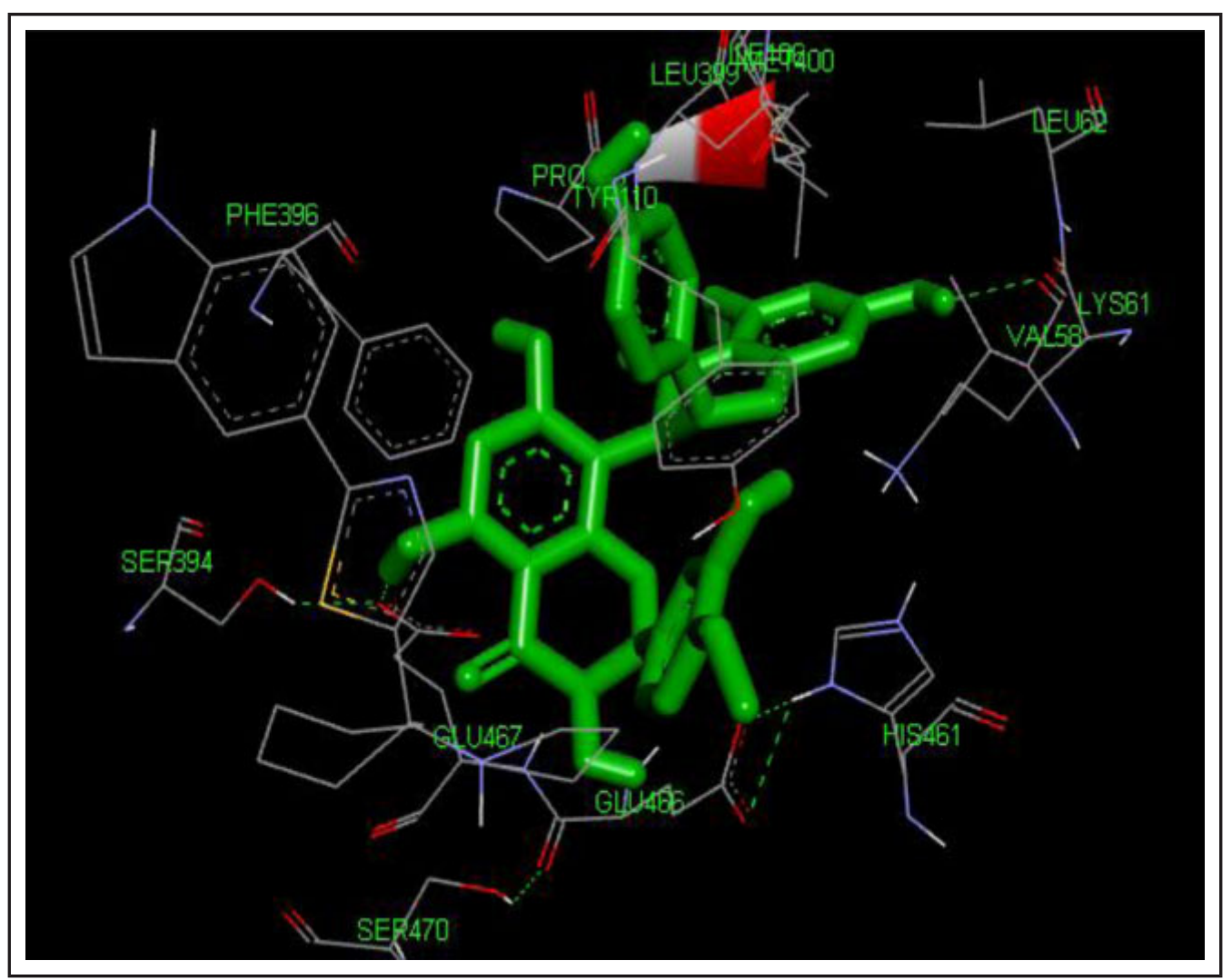

Figure 4. 2D Schematic presentation of the active site of Trypanothione reductase and ligand (Kolaviron).

\section{Effects of Chrysin and Kolaviron on Trypanothione Reductase in vitro}

Table 2 shows the result of the in vitro assay of the enzyme trypanothione reductase (from $T$. congolense lysate) isolated from the blood stream form of the parasite. The highest \% inhibition for the compounds was found to be $66.78 \%$ and $53.80 \%$ for Chrysin and Kolaviron, respectively (Figure 5 and Table 2). The calculated $I C_{50}$ values for Chrysin and Kolaviron were 1.86 and $2.64 \mu \mathrm{g} / \mathrm{ml}$, respectively. This qualified Chrysin and Kolaviron to be potent having inhibited the enzyme activity half way, thus, we proceeded with the in vivo studies.

\section{Effects of Chrysin and Kolaviron on Parasitaemia and Trypanothione Reductase in vivo}

Figure 6 shows the result of daily parasitaemia estimation of $T$. congolense infection in male Wistar rats with a post infection treatment from day 5 . Relative decrease in the parasitaemia level was found after day 12 of the treatment. At the end of the treatment, the number of organisms per $\mathrm{ml}$ of blood was 256 in Chrysin and 316 in Kolaviron treated groups relative to 2483 of the non-treated but infected group. To ascertain whether the observed anti-trypanosomal activities of these compounds, have effects on the activity of trypanothione reductase in vivo, we checked for its activity upon treatment with the said compounds.

Table 3 shows the result of the in vivo \% inhibition of the compounds on the enzyme. Upon administration of the compounds to the infected Wistar rats for the period of 12 days, Chrysin and Kolaviron were found to inhibit the activity of the enzyme by $57.67 \%$ and $46.90 \%$, respectively. Interestingly, the \% inhibition was lower compared that of in vitro which may likely be because of some metabolic
Table 2. \% Inhibition of Chrysin and Kolaviron against Trypanothione Reductase from $T$. congolense in vitro

\begin{tabular}{ccccc}
\hline S/No & Compound & $\begin{array}{c}\text { Final } \\
\text { concentration } \\
(\mu \mathrm{g} / \mathrm{ml})\end{array}$ & $\begin{array}{c}\text { Mean } \% \\
\text { Inhibition }\end{array}$ & $\begin{array}{c}\mathrm{IC}_{50} \\
(\mu \mathrm{g} / \mathrm{ml})\end{array}$ \\
\hline 1. & Chrysin & 0.38 & 6.28 & \\
& & 0.77 & 18.96 & 1.86 \\
& 1.54 & 43.59 & \\
& & 3.08 & 66.78 & \\
& & & & \\
& Kolaviron & 0.38 & 4.96 & \\
& & 0.77 & 15.77 & \\
& & 1.54 & 36.68 & \\
& & 3.08 & 53.80 & \\
\hline
\end{tabular}

activities. Thus, one could see that the observed effects were basically at the fine not a coarse type level. We therefore went further to determine the effects of these compounds on the expression of trypanothione reductase gene in vivo.

\section{Effects of Chrysin and Kolaviron on the Expression of Trypanothione} Reductase in vivo

As depicted in Figure 7, upon administration of Chrysin and Kolaviron to the rats infected with $T$. congolense, the relative change in fold was found to be 1.352 and 0.642 in Chrysin and Kolaviron treated groups respectively, relative to untreated but infected group $(P<0.05)$ with 3.530 -fold increase in expression. This may as well indicate down regulating effects on the part of Chrysin and Kolaviron. 


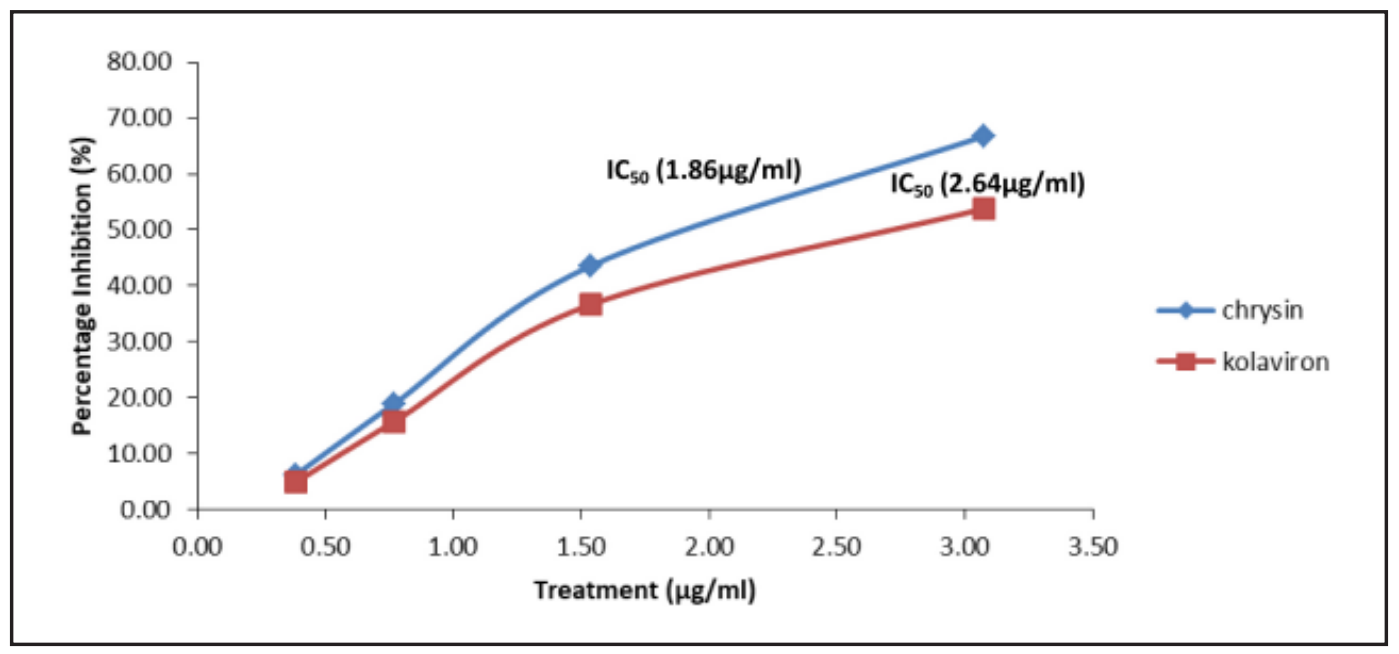

Figure 5. \% inhibition of Kolaviron and Chrysin against Trypanothione Reductase from T. congolense in vitro.

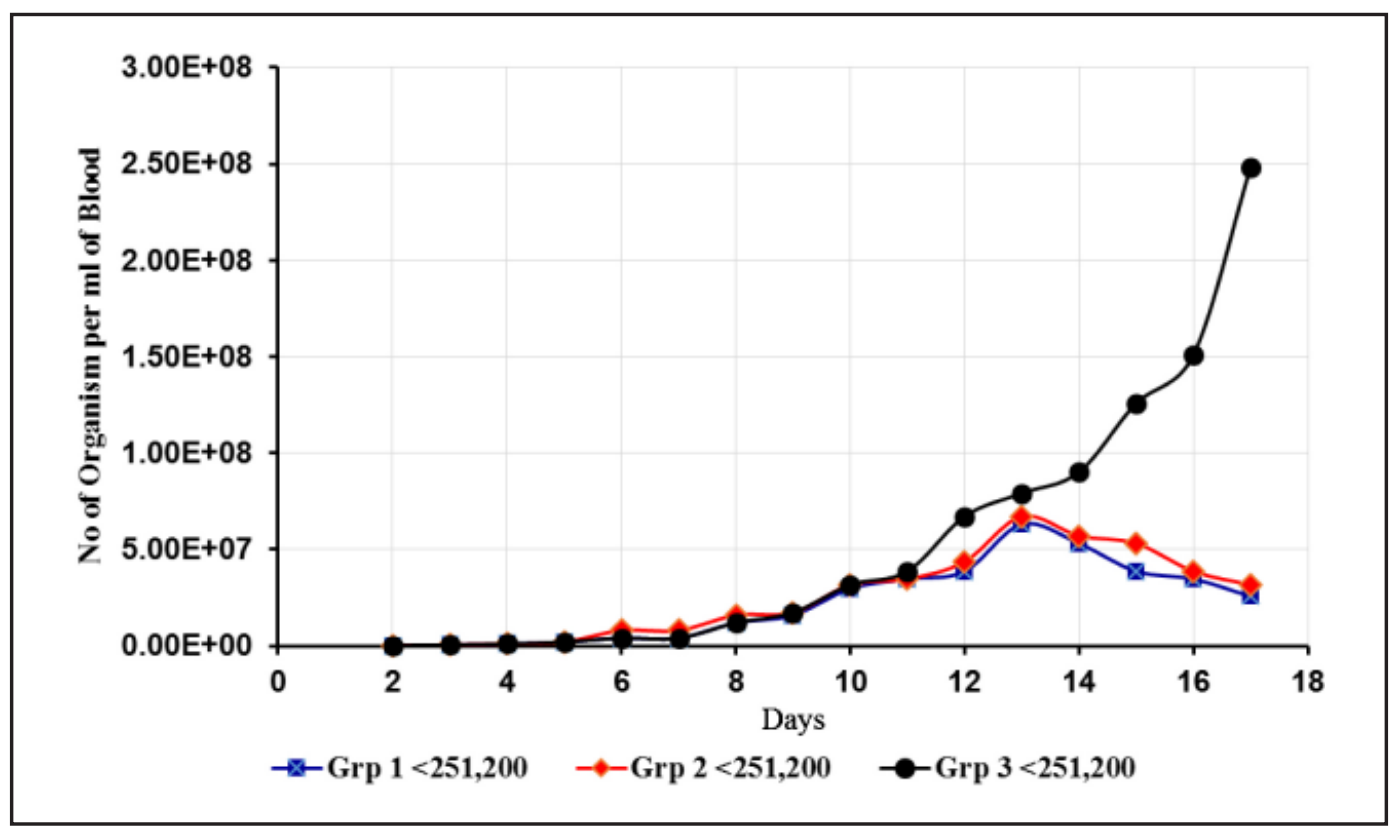

Figure 6. Daily Parasitaemia before and after treatment with Kolaviron and Chrysin in $T$. congolense infected Wistar rats. Group 1 (Grp 1): Infected and treated with Chrysin (40mg/Kg/b.w.), Group 2 (Grp 2): Infected and treated with Kolaviron (200mg/Kg/b.w.), Group 3 (Grp 3): Infected untreated.

Table 3. \% Inhibition of Chrysin and Kolaviron against Trypanothione Reductase in Trypanosoma congolense infected Wistar Rats

\begin{tabular}{cccc}
\hline S/No & Compound & $\begin{array}{c}\text { Treatment } \\
\text { (mg/Kg/b.w.) }\end{array}$ & $\begin{array}{c}\text { Mean \% } \\
\text { Inhibition }\end{array}$ \\
\hline 1. & Chrysin & 40 & 57.67 \\
2. & Kolaviron & 200 & 46.90 \\
\hline
\end{tabular}

\section{DISCUSSION}

Trypanosomiasis is a deadly disease caused by extracellular parasites (Trypanosoma) which are spread by tsetse flies (Genus Glossina) (Philippe et al., 2017). Currently, the treatment of these diseases depends on only a handful of drugs are available and their efficacy had recently suffered from extensive drug resistance and grave side effects (Baker et al., 2013). Thus, there is an urgent need to discover new compounds as starting point for the development of potent drugs which are efficient with less side effects, preferably meddling with exclusive crucial pathways of these parasites (Beig et al., 2015). One of the urgencies in tropical medicine research has been the identification and characterisation of parasite-specific biomolecules, which play pertinent physiological starring role and in consequence might be subjugated as selective targets for drug discovery and development (Tovar et al., 2006). One of these targets is trypanothione reductase (TryR) which is an enzyme responsible for maintaining the redox balance in this protozoan parasite. The absence of TryR from the mammalian host and the sensitivity of trypanosomatids to oxidative stress make TryR a striking target for Trypanosomiasis therapeutics (Saha \& Sharma, 2014). In this study, we report 


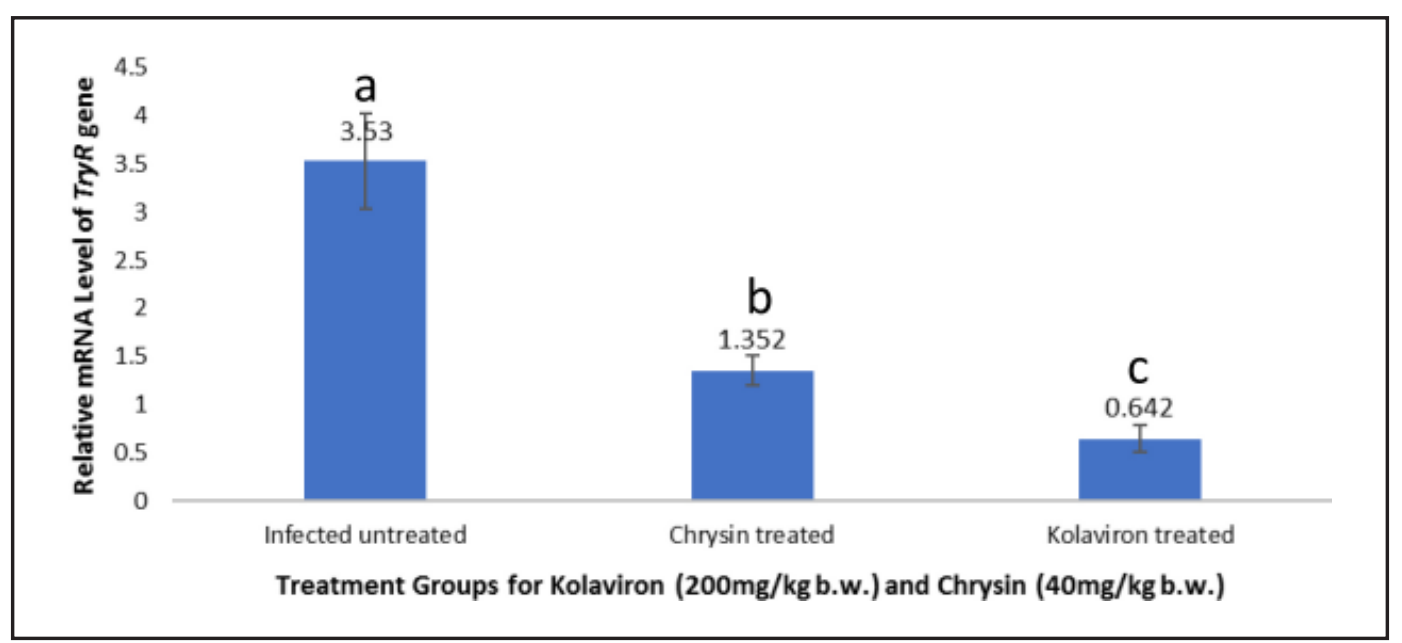

Figure 7. Effect of Chrysin and Kolaviron administration on Trypanothione reductase Expression in the blood stream form of $T$. Congolense in Wistar rats. Means (bars) with different superscript are statistical significant $(p<0.05)$.

for the first time the use of Chrysin and Kolaviron via in silico, in vitro and in vivo screening against trypanothione reductase in Trypanosoma congolense infection.

The results of the molecular modelling of the two compounds against trypanothione reductase (TryR) were in clear agreement with the report of Chiu et al. (Chiu et al., 2008) that TryR has a hydrophobic active site which tolerates non-cognate substrate architecture as such non-reducible acyclic and cyclic substrate analogues display inhibition against it. The tricyclic moiety of these compounds was shown to lodge against the hydrophobic wall of TryR active site formed by Trp21 and Met113, with the aminopropyl side chain pointing towards the Glu466' and Glu467' residues.

The results of in vitro study demonstrate the potency of Kolaviron and Chrysin as TryR inhibitors judging by the calculated $I_{50}$ values. This by implication indicates the ability of Chrysin and Kolaviron to decrease the rate of the conversion of trypanothione disulphide to trypanothione by binding at the polyamine moiety in the hydrophobic pocket of the enzyme as previously described (Beig et al., 2015). The alteration of the binding of trypanothione disulphide to TryR increases the accumulation of free radicals in the cell with resultant increase in oxidative stress in the parasite cell with an ultimate cell death as previously reported (Kumar et al., 2014; Saravanamuthu et al., 2004). This may as well explain the observed decrease in parasitaemia due to these compounds.

Administration of Kolaviron and Chrysin led to decrease in parasitaemia in the treated groups as compared to the untreated, though not completely eliminated. This may invariably corroborate with the observed inhibition of the said activity of the enzyme thereby reducing the proliferation of the parasite as a result of an increased oxidative stress leading to cell death as previously reported (Richardson et al., 2009). Chrysin has also been reported against Trypanoma brucei previously (Baldim et al., 2017), which have further validated our finding. Accordingly, the results of the in vivo \% inhibition of the compounds on the enzyme have shown the potency of the compounds corroborating the in vitro findings.

Going further, from the gene expression analysis, administration of Kolaviron and Chrysin drastically down regulated the expression of TryR in vivo. By inference, the effects of these compounds may be nucleic acid specific in addition to their effect at the fine type level of TryR activity. Flavonoids administrations were implicated in affecting the activity of TryR due to their wide range of biological activities (Dixit, 2014). These effects may likely go down to molecular level as clearly for the first time depicted in the current study, as flavonoids have recently demonstrated in vivo and in vitro leishmanicidal, trypanocidal, antioxidant, and pro-oxidant properties (Tasdemir et al., 2006; Baldim et al., 2017).

The findings from this study for the first time, has clearly shown the potency of Kolaviron and Chrysin as inhibitors of TryR at the fine and coarse type levels. This may have unraveled the potency of these compounds in the management of trypanosome infection as it relates with the role of TryR as parasite protective agent. We recommend further studies on the kinetics of inhibitions by these flavonoids vis-à-vis their synergistic effects.

\section{ACKNOWLEDGEMENTS}

We appreciate the Head, Department of Biochemistry, Ahmadu Bello University Zaria, Kaduna State, Nigeria. We also appreciate the efforts of Dr. Balogun Emmanuel of the Department of Biochemistry, Ahmadu Bello University Zaria, and the entire team of Africa Centre of Excellence for Neglected Tropical Diseases and Forensic Biotechnology (ACENTDFB).

\section{Conflict of interest}

None declared.

\section{Funding}

This work was fully funded by Africa Centre of Excellence for Neglected Tropical Diseases and Forensic Biotechnology (ACENTD FB), Ahmadu Bello University, Zaria, Kaduna State, Nigeria.

\section{REFERENCES}

Adaramoye, O.A. \& Arisekola, M. (2013). Kolaviron, a biflavonoid complex from Garcinia kola seeds, ameliorates ethanol-induced reproductive toxicity in male wistar rats. Nigerian Journal of Physiological Sciences 28: 9-15.

Bailey, S., Smith, K., Fairlamb, A.H. \& Hunter, W.N. (1993). Substrate interactions between trypanothione reductase and W-glutathionylspermidine disulphide at $0.28-\mathrm{nm}$ resolution. European Journal of Biochemistry 75: 67-75. https://doi.org/10.1111/j.1432-1033.1993.tb17734.x 
Baker, N., de Koning, H.P., Mäser, P. \& Horn, D. (2013). Drug resistance in African trypanosomiasis: The melarsoprol and pentamidine story. Trends in Parasitology 29(3): 110118. https://doi.org/10.1016/j.pt.2012.12.005

Baldim, J.L., Gonçalves, B., Alcântara, V. De, Domingos, S., Soares, M.G., Caldas, I.S. \& Chagas-paula, D.A. (2017). The Correlation between Chemical Structures and Antioxidant, Prooxidant, and Antitrypanosomatid Properties of Flavonoids. Oxidative Medicine and Cellular Longevity 2017: 3789856. https://doi.org/10.1155/2017/3789856

Baral, T.N. (2010). Immunobiology of African trypanosomes: Need of alternative interventions. Journal of Biomedicine and Biotechnology 2010: 389153. https://doi.org/10.1155/ 2010/389153

Barrett, M.P., Boykin, D.W., Brun, R. \& Tidwell, R.R. (2007). Human African trypanosomiasis/: pharmacological reengagement with a neglected disease. British Journal of Pharmacoclogy 2005(153): 1155-1171. https://doi.org/ 10.1038/sj.bjp.0707354

Beig, M., Oellien, F., Garoff, L., Noack, S. \& Krauth-, R.L. (2015). Trypanothione Reductase/: A Target Protein for a Combined In Vitro and In Silico Screening Approach. Plos Neglected Tropical Diseases 9: e0003773. https://doi.org/ 10.1371/journal.pntd.0003773

Chanie, M., Adula, D. \& Bogale, B. (2013). Socio-economic assessment of the impacts of trypanosomiasis on cattle in girja district, southern oromia region, southern ethiopia. Acta Parasitology Globalis 4: 80-85. https://doi.org/ 10.5829/idosi.apg.2013.4.3.7523

Chiu, J., Khan, Z.A. \& Farhangkhoee, H.C.S. (2008). Curcumin prevent diabetes-associated abnormalites in kidney by inhibiting p300 and nuclear factor-kappaB. Nutrition 92(1): 964-972. https://doi.org/10.1016/j.nut.2008.12.007

Dixit, S. (2014). Anticancer Effect of Rutin Isolated from the Methanolic Extract of Triticum aestivum Straw in Mice. Medical Sciences 2(4): 153-160. https://doi.org/10.3390/ medsci2040153

Farombi, E.O., Adedara, I.A., Ajayi, B.O., Ayepola, O.R. \& Egbeme, E.E. (2013). Kolaviron, a Natural Antioxidant and Anti-Inflammatory Phytochemical Prevents Dextran Sulphate Sodium-Induced Colitis in Rats. Basic and Clinical Pharmacology and Toxicology 113(1): 49-55. https://doi.org/ 10.1111/bcpt.12050

Gupta, N., Chauhan, R.S. \& Pradhan, J.K. (2014). Rutin: A bioactive flavonoid. In Handbook of Medicinal Plants and their Bioactive Compounds 661: 51-57.

Herbert, W.H. \& Lumsden, W.R. (1976). Rapid matching method. Eperimental Parasitology 431(40): 427-431.

Holloway, G.A., Charman, W.N., Fairlamb, A.H., Brun, R., Kaiser, M., Kostewicz, E. \& Baell, J.B. (2009). Trypanothione Reductase High-Throughput Screening Campaign Identifies Novel Classes of Inhibitors with Antiparasitic Activity. Antimicrobial Agents and Chemotherapy 53(7): 2824-2833. https://doi.org/10.1128/AAC.01568-08

Kaidama, W.M. \& Gacche, R.N. (2015). Anti-Inflammatory Activity of Chrysin in Acute and Chronic Phases of Inflammation in Guinea Pigs. International Journal of Scientific and Research Publication 5(2): 1-5.

Kumar, S., Ali, R. \& Bawa, S. (2014). Mini review on tricyclic compounds as an inhibitor of trypanothione reductase. Journal of Pharmacy and Bioallied Sciences 6(4): 222-229. https://doi.org/10.4103/0975-7406.142943
Malele, I., Craske, L., Knight, C., Ferris, V., Njiru, Z., Hamilton, P. \& Gibson, W. (2003). The use of specific and generic primers to identify trypanosome infections of wild tsetse flies in Tanzania by PCR. Infection, Genetics and Evolution 3(4): 271279. https://doi.org/10.1016/S1567-1348(03)00090-X

Muhammad, A., Funmilola, A., Aimola, I.A., Ndams, I.S., Inuwa, M.H. \& Nok, A.J. (2017). Kolaviron shows anti-proliferative effect and down regulation of vascular endothelial growth factor- $\mathrm{C}$ and toll like receptor- 2 in Wuchereria bancrofti infected blood lymphocytes. Journal of Infection and Public Health 10(5): 661-666. https://doi.org/10.1016/j.jiph.2017. 05.006

Olaleye, S.B., Farombi, E.O., Adewoye, E.A., Owoyele, B.V., Onasanwo, S.A. \& Elegbe, R. (2000). Analgesic and AntiInflammatory Effects of Kolaviron (a Garcinia kola Seed Extract). African Journal of Biomedical Research 3: 171-174.

Peacock, L., Cook, S., Ferris, V., Bailey, M. \& Gibson, W. (2012). The life cycle of Trypanosoma (Nannomonas) congolense in the tsetse fly. Parasite (Paris, France) 5(109): 1-13.

Philippe, B., Giuliano, C., Vincent, J. \& Gerardo, P. (2017). Human African trypanosomiasis. The Lancet 6736(17): 23972409. https://doi.org/10.1016/S0140-6736(17)31510-6

Richardson, J.L., Nett, I.R.E., Jones, D.C., Abdille, M.H., Gilbert, I.H. \& Fairlamb, A.H. (2009). Improved Tricyclic Inhibitors of Trypanothione Reductase by Screening and Chemical Synthesis. Chemmedchem 4: 1333-1340. https://doi.org/ 10.1002/cmdc. 200900097

Saha, D. \& Sharma, A. (2015). Docking-based screening of natural product database in quest for dual site inhibitors of Trypanosoma cruzi trypanothione reductase (TCTR). Medicinal Chemistry Research 24: 316-333. https://doi.org/ 10.1007/s00044-014-1122-x

Saravanamuthu, A., Vickers, T.J., Bond, C.S., Peterson, M.R., Hunter, W.N. \& Fairlamb, A.H. (2004). Two interacting binding sites for quinacrine derivatives in the active site of trypanothione reductase: A template for drug design. Journal of Biological Chemistry 279(28): 29493-29500. https:/ /doi.org/10.1074/jbc.M403187200

Tasdemir, D., Kaiser, M., Brun, R., Yardley, V., Schmidt, T.J., Tosun, F. \& Ru, P. (2006). Antitrypanosomal and Antileishmanial Activities of Flavonoids and Their Analogues/: In Vitro, In Vivo, Structure-Activity Relationship, and Quantitative Structure-Activity Relationship Studies. Antimicrobial Agents and Chemotherapy 50(4): 1352-1364. https://doi.org/10.1128/AAC.50.4.1352-1364.2006

Tovar, J., Fonseca, S.G., Moraes, R.H., Cruz, A.K., Hothersall, J.S., Ferreira, S.H. \& Medicina, F. De. (2006). Glutathione and the redox control system trypanothione / trypanothione reductase are involved in the protection of Leishmania spp. against nitrosothiol-induced cytotoxicity. Brazzillian Journal of Medical and Biological Research 39: 355-363. https://doi.org/10.1590/s0100-879×2006000300006

Umar, I.A., Ibrahim, M.A., Fari, N.A., Isah, S. \& Balogun, D.A. (2010). Extracts from different parts of Khaya senegalensis. Journal of Cell and Animal Biology 4: 91-95. 